\title{
Performance Investigation of VoIP Over Mobile WiMAX Networks through OPNET Simulation
}

\author{
Ilyas Khudhair Yalwi Dubi ${ }^{1}$, Ravie Chandren Muniyandi ${ }^{2}$ \\ Centre for Cyber Security Faculty of Information Science \& Technology \\ Universiti Kebangsaan Malaysia, Bangi, Malaysia
}

\begin{abstract}
Worldwide Interoperability for Microwave Access (WiMAX) is regarded as a promising technology that can provide wireless communication because of its advantages which include, high-speed data rates, high coverage and low cost of development and maintenance. WiMAX also supports the performance of Voice over Internet Protocol (VoIP), which is expected to replace conventional circuit switched voice services. VoIP requires to accurately design of QoS configurations over WiMAX networks. This paper focuses on studying and analyzing the performance of VoIP over WiMAX mobile networks. WiMAX network and VoIP technology such as mobility, WiMAX service classes, number of nodes and VoIP codecs are studied and analyzed. WiMAX network is simulated in a different manner using the simulation program known as OPNET Modeler. Simulation results established that the service layer Unsolicited Grand Service (UGS) is more appropriate for VoIP service because it has the best standard and performance. It was also observed that the least delay and highest value of customer satisfaction rate of services is demonstrated by the G.723.1 best coding. It also maintains the minimum consumption of capacity.
\end{abstract}

Keywords-Voice over Internet Protocol (VoIP); R-score; Worldwide Interoperability of Microwave Access (WiMAX); quality of service (QoS); OPNET 14.5

\section{INTRODUCTION}

Great investments have been made by the telecommunications into developing and deploying mobile Worldwide Interoperability for Microwave Access (WiMAX) networks due to the high demand of broadband wireless access by mobile users [1]. The aim of this investment is to meet the needs of mobile users. In this regard, Voice over Internet Protocol (VoIP) has been identified as one of the appropriate applications that can facilitate the deployment of mobile WiMAX networks [2]. The amount of voice traffic in these networks can be increased as a result of the legal desire for bundling voice and data. Thus, a major application that can be used in mobile WiMAX networks is VoIP, which is currently the technology used for voice call through packet switch networks [3]. The increase of VoIP applications such as Skype, Google Talk, WhatsApp etc. alongside the emergence of mobile WiMAX networks deployment, has made VoIP over WiMAX an attractive market [4]. More so, this increase of VoIP applications has been a motivation for both equipment suppliers and carriers to initiate the next trend in telecommunication innovation [5-7]. Despite the benefits of VoIP over WiMAX networks, it is accompanied by some challenges. Efforts have been made by researchers to address some these challenges by enhancing the hardware of the application, but these efforts have remained insufficient [810]. Some of these challenges are associated with the delivery of voice communication and multimedia session over the internet. This implies that, it is important to select the appropriate network environment that can enable the delivery of multimedia session and voice communication over the internet. The optimization of the capacity of VoIP call over WiMAX networks is one major problem [11]. This research is carried out in order to analyze the performance of VoIP over mobile WiMAX networks.

The presence of WiMAX networks that provide long range coverage has made wireless internet abundantly available. The implication of this availability is that, portable devices that run VoIP operating on WiMAX have the potentials of gaining more popularity than cellular phones [12]. However, the WiMAX networks are limited by the fact that applications operating on them could be affected by the physical phenomena of the wireless transmission medium [13]. More so, with wireless networks, the quality of real-time applications, especially VoIP are often affected by network problems like packet delay and loss. It is based on this, that this study is conducted in order to carry out an analysis of the performance of VoIP applications by studying the mobile WiMAX. This paper aims at studying and analyzing the performance of VoIP over mobile WiMAX networks.

Organization of this paper is listed as follows: In Section 2, we provide a brief overview of WiMAX. In Section 3, 4 deployments of different scenarios of WiMAX network for the purpose of studying and analyzing the performance of VoIP. we present the simulation model and results. Section 5 Future Work. Conclusions are drawn in Section 6.

\section{PRevious Studies}

Recently, there has been a rapid growth in the different wireless technologies, thereby leading to increasing demand for wireless data services and multimedia applications like VoIP, video and audio streaming [18]. This demand has led to growing research interest in both VoIP systems and wireless technologies so as to meet these user demands while providing quality service. The popularity of VoIP, particularly for the deployment of mobile WiMAX is increasing globally [12]. Researchers have focused on different areas of VoIP over WiMAX. Some of the research carried out are discussed in this section. Adhicandra [13] focused on investigating the WiMAX network in terms of data and voice support. He examined the deployment of QoS over WiMAX network, and 
compared the performance results obtained through the use of two different WiMAX service classes (UGS and ertPS). In another study [14], a fixed WiMAX network was considered with the aim of evaluating the performance of VoIP. In this study, an assessment of the different transmission schemes in terms of packet rate, Mean Opinion Score (MOS), cumulative good-put and sample loss rate were carried out. In [15], a traffic-aware scheduling algorithm was proposed for VoIP applications in WiMAX networks. More so, they conducted an evaluation of their proposed method and made a comparison of it with other traditional methods. In addition to this, they provided a discussion on the trade-off between delay and bandwidth efficiency. It was observed that through the use of the proposed method of scheduling, the efficiency of VoIP over WiMAX is enhanced.

Furthermore, a discussion on the wide range of issues associated with VoIP and models of measuring voice quality was also done in [16]. In this study, a novel methodology that can be used for the prediction of voice quality in a manner that is not intrusive was outlined. Carvalho et al. [17] focused on the area of voice quality with the main aim was to design a tool for measuring voice quality through the use of ITU-T Emodel [19]. The tool was tested using some calls generated from two endpoints at different cities in Brazil. Jadhav et al. [20], performed an extensive simulation with the main aim of evaluating the performance of WiMAX and UMTS (Universal Mobile Telephone System) for the facilitation of VoIP traffic. Results of the simulation revealed that WiMAX is better than UMTS with an adequate margin. It was also found that in comparison to UMTS, the WiMAX is more appropriate for VoIP applications.

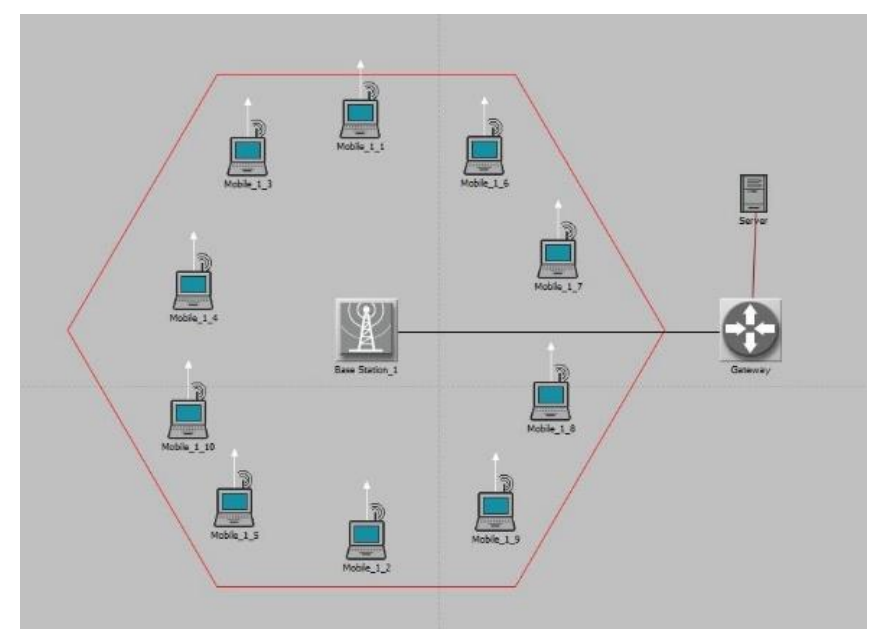

Fig. 1. WiMAX Network Model.

TABLE I. SIMULATION PARAMETERS

\begin{tabular}{|l|l|}
\hline Parameter & Value \\
\hline Bandwidth & $20 M H z$ \\
\hline Duplex Mode & $T D D$ \\
\hline QoS Service & UGS, rtPS \& BE \\
\hline Voice Codec & G.711, G.723.1\& G.729A \\
\hline Application & VoIP \\
\hline
\end{tabular}

\section{METHODOLOGY}

In this paper, quantitative approaches were used. Firstly, a comprehensive review of literature was done; the review involved investigating the case studies and ethnographies. Afterwards, attention is paid to the problem and possible solutions. One of the popularly used research methodology for studying and analyzing the performance of wireless and wired networks is computer simulation. In our studies, we employed the use of OPNET Modeler [21]. The use of OPNET in this study enabled the deployment of different scenarios of WiMAX network for the purpose of studying and analyzing the performance of VoIP. Simulation study was carried out to evaluate the performance of VoIP over the WiMAX networks. Different parameters such as jitter, MOS value, packet end-toend delays and, packets sent and received, were used to measure the performance of VoIP over WiMAX. The purpose of deploying different scenarios is to address different aspects of the WiMAX network such as load, data traffic, available bandwidth, network capacity, QoS classes and mobility, which may have an effect on VoIP traffic. For each of the scenarios, many simulation runs were performed considering the following issues: initial variables values, runs length, model, generation of random numbers and settle-down time. The outputs of simulations were obtained and statistically analyzed. Afterwards, the simulation results were examined, interpreted and graphically presented. Recommendations have been provided based on the simulation results. The purpose of providing the recommendations is to address the problem statement of the paper. The WiMAX network model is made up of one cell with a set radius of 5 kilometers, and an IP backbone, which contains just one Voice Server. The cell contains a single Base Station and many Mobile Stations subject to the simulation scenario. The WiMAX network model that is considered in the simulations is illustrated in Fig. 1. The general parameters of the WiMAX network model are presented in Table 1.

\section{RESUlTS AND DISCUSSION}

The outputs of the different simulation runs which have been obtained were statistically analyzed. The simulation results are: throughput, delay, jitter and mean opinion score (MOS). The effect of the configuration of mobile WiMAX network on the performance of VoIP is determined through the first three results. Furthermore, the quality of VoIP call is measured through the last result which is MOS. In the next section the results which have been obtained, are graphically presented and discussed.

\section{A. Scenario (1)}

The purpose of designing Scenario (1) is to identify the ideal WiMAX service class which facilitates the best VoIP performance. The different service classes were compared by representing the data that have been gathered from the three service classes on the one chart. This is the easiest way to observe the behavior of various QoS parameters for the same traffic over diverse kinds of services classes. The comparative plots can be seen from Fig. 2 to Fig. 5.

Fig. 2 is a single chart that illustrates the throughput for all three service classes. Among the three classes, the throughput 
for UGS flow is the highest. This can be attributed to the fact that constant bit rate traffic is considered during the designing of UGS service class. The MS is able to forward more data because of the periodic bandwidth which is allocated to it by the BS. The result revealed that the throughput for rtPS traffic is better than BE. More so, it was observed that a decrease begins to occur in the throughput when the number of nodes goes beyond 8 .

The Mean Opinion Score obtained for the three service classes is presented in Fig. 3. Out of the three classes, the highest MOS value was scored by the UGS service. The MOS value of rtPS is slightly better that that of BE. It was also observed that an increase in the number of nodes cause the MOS value to decrease.

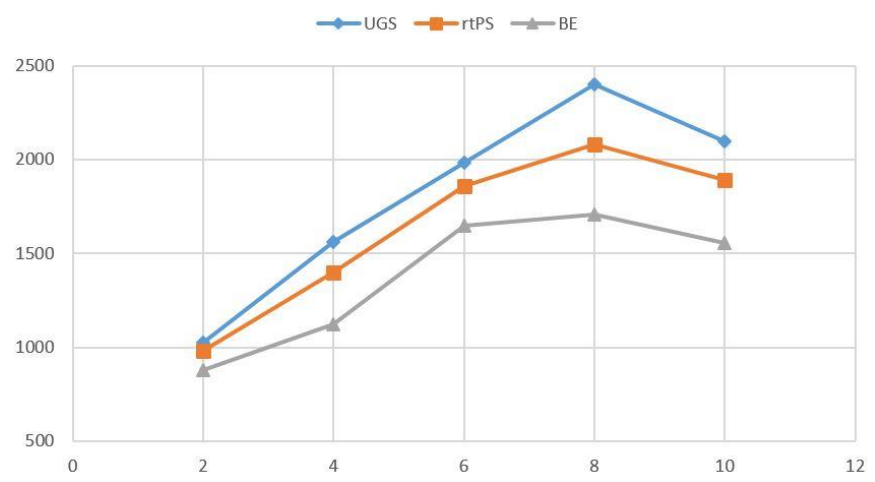

Fig. 2. Throughput for Different Service Classes.

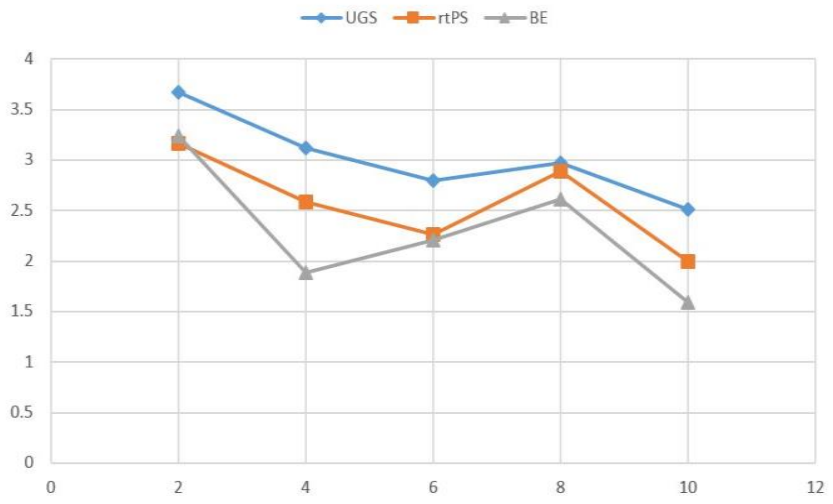

Fig. 3. MOS for Different Service Classes.

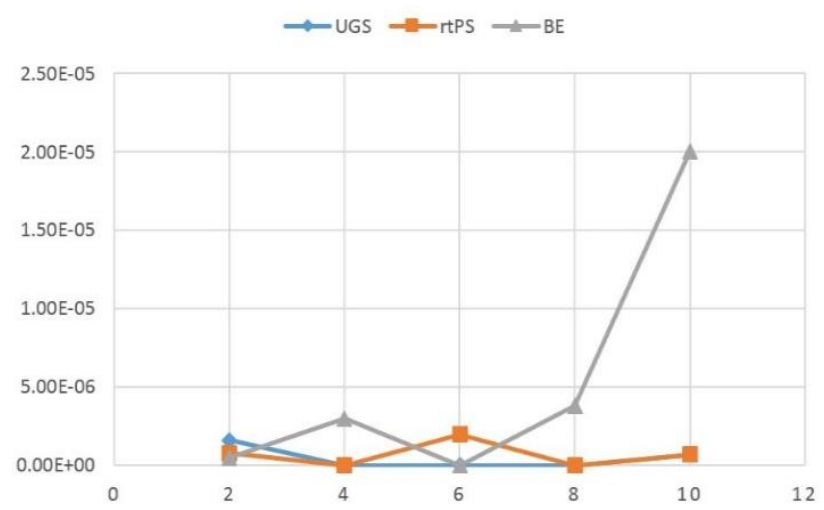

Fig. 4. Jitter for Different Service Classes.

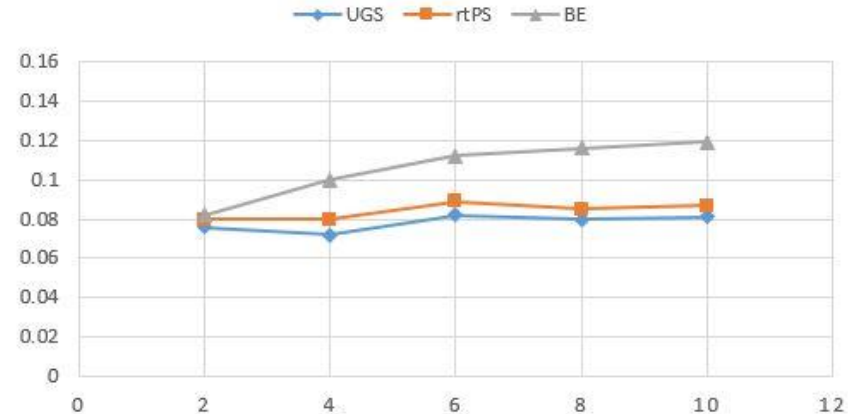

Fig. 5. DE2E for Different Service Classes.

Fig. 4 shows the average jitter for all the three service classes. In order to the comparison easy, the graph is drawn on the logarithmic scale. The highest jitter was recorded for $\mathrm{BE}$ service class. It was also found that there is not much variation in the average jitter for UGS service class as an increase occurs in the number of nodes. Secondly, the value is very small. Based on the results, the jitter values for rtPS were low and falls very close to UGS. One of the major parameters for measuring the perceived quality of voice as it arrives the destination node is jitter. Fig. 5 shows the end-to-end delay for the three service classes. The difference between the values for UGS and rtPS service classes was very minimal without exceeding $0.09 \mathrm{~s}$ irrespective of the number of nodes. Conversely, the highest delay was recorded for BE service class; with an increase in the number nodes, the delay increase as well.

Conclusively, it was observed that the highest throughput, bets MOS value, lowest jitter and delay were demonstrated by UGS service class. This makes it the most suitable candidate for VoIP traffic. The results of simulation confirm that the UGS service class is capable of handling fixed sized packets that are generate at a regular interval.

\section{B. Scenario (2)}

As revealed by the results of Scenario (1), UGS is the most suitable for WiMAX service class for VoIP traffic. Scenario (2) was been designed to run under the UGS service class while analyzing the performance of VoIP using diverse voice codecs (G.711, G.723.1 and G.729A). A single chart is used in presenting all the data obtained from all codecs. This is the easiest way to observe the performance of VoIP traffic over various kinds of codecs. The comparative plots are presented in Fig. 6 through Fig. 9 show the comparative plots.

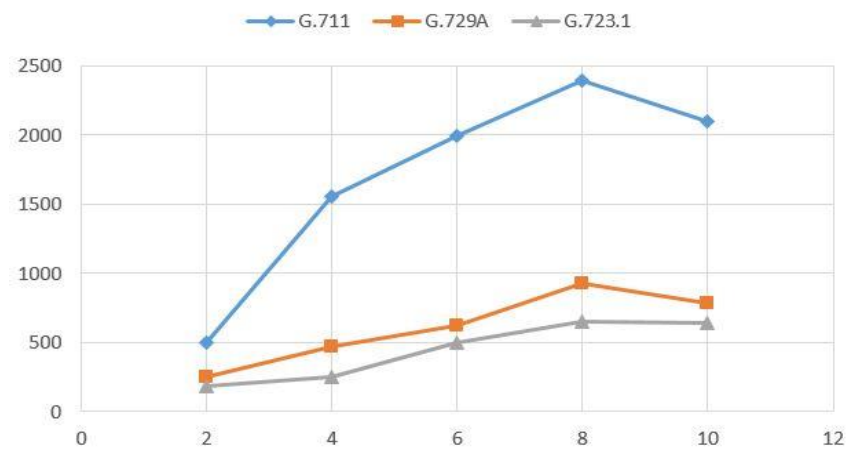

Fig. 6. Throughput for Different VoIP Codecs. 


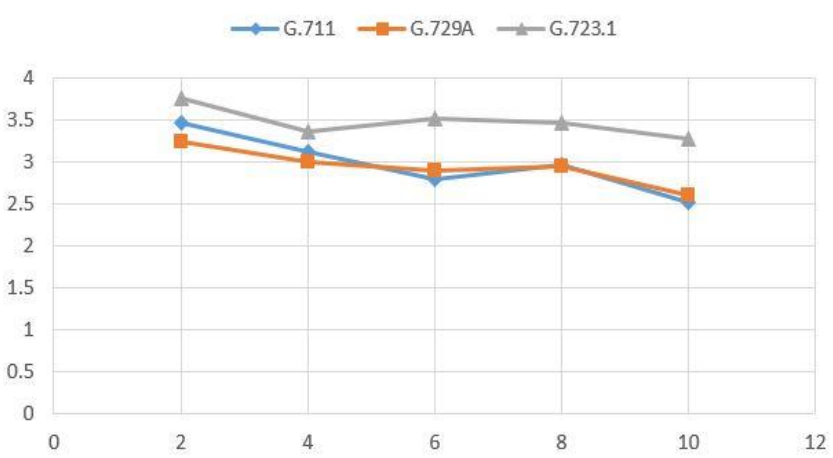

Fig. 7. MOS for Different VoIP Codecs.

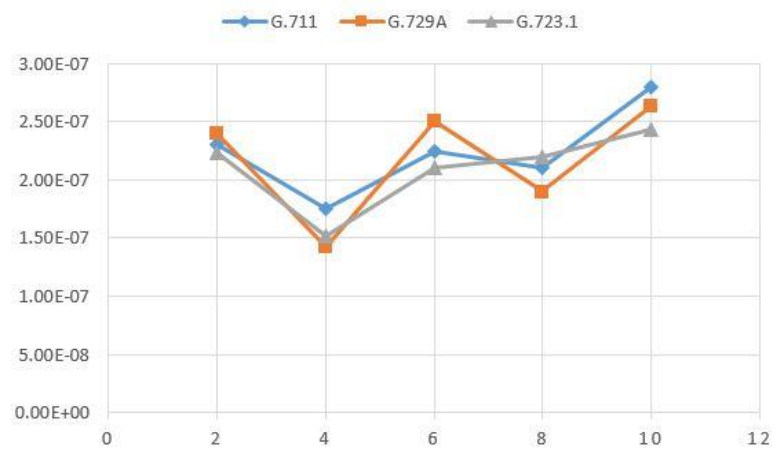

Fig. 8. Jitter for Different VoIP Codecs

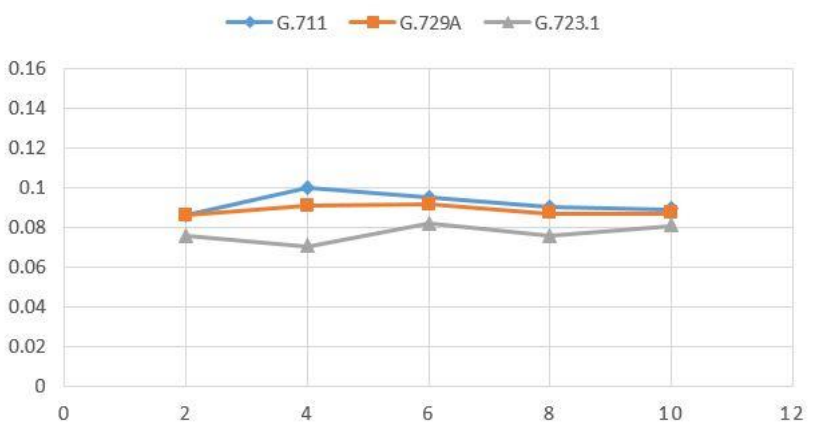

Fig. 9. DE2E for Different VoIP Codecs.

Fig. 6 shows the throughput for the different codecs. As expected, the highest throughput was achieved by G.711 codec because of its high bandwidth consumption. The next higher throughput is for G.729A, while the lowest throughput was recorded for the G732.1 codec.

In Fig. 7 the Mean Opinion Score (MOS) values for all the three codecs are presented. Among the three classes, the best MOS value was demonstrated by the G.723.1 codec. The MOS values of G.711 and G.729A codecs were roughly.

Fig. 8 shows the jitter across different VOIP codecs. Results revealed small values with insignificant pattern. For all practical purposes, the average jitter for all codecs is insignificant and negligible. Fig. 9 shows the end-to-end delay across different VOIP codecs. It can be observed that the maximum delay was demonstrated by G711, while the G723.1 demonstrated minimum delay. There is a small range of variation in the values, with insignificant pattern of variation caused by an increase in the number of nodes. In conclusion, it is observed that the overall performance characteristics of G.723.1 codec is better than that of the other two codecs, with G.723.1 codec scoring the best MOS value and lowest delay coupled with less consumption of bandwidth.

\section{FUTURE WORK AND CONCLUSION}

Through our study of VoIP performance analysis based on the study of investigation of WiMAX service classes and VoIP codecs. We would like to refer to some suggestions that improve the performance study: seeing for different traffic types (VoD, FTP, HTTP, etc...), Study of ertPS for VoIP with silence suppression. And Consideration for other mobile WiMAX aspects (mobility patterns/speeds, handoff, large number of mobile stations, transmission power, cell radius, etc...). WiMAX continues to advance, and a new standard is being developed. The standard is IEEE $802.16 \mathrm{~m}$ and aims for $1 \mathrm{Gbps}$ for nomadic and $100 \mathrm{Mbps}$ for Mobile terminals. This is a revolution in the field of mobile communications, which will be fruitful to achieve further research on this standard.

In this paper, the research involved simulation studies for the purpose of analyzing the performance of VoIP over mobile WiMAX networks. This was achieved, by investigating WiMAX QoS service classes (UGS, rtPS \& BE) and VoIP codecs (G.711, G.729 \& G.723.1). The performance was analyzed in terms of critical parameters like MOS, throughput, end-to-end delay and jitter. The use of OPNET Modeler 14.5A was employed in the computer simulation that produced the results. The results of the simulation revealed that UGS service class is more suitable for VoIP as it demonstrated the best performance. UGS service class is capable of handling real-time service flows that produce fixed size packets at a regular interval, which is the case for VoIP. In addition, the results showed that the G.723.1 demonstrated lower delay with higher MOS and minimal consumption of bandwidth, thereby making it better than codecs G.711 and G.729A. Overall, this paper gave a good insight into the technical details of WiMAX while learning the intricacies of the OPNET simulator.

\section{ACKNOWLEDGMENT}

This study was supported by the Ministry of Education, Government of Malaysia under the fundamental research grant: FRGS/1/2015/ICT04/UKM/02/3.

\section{REFERENCES}

[1] Aguado, M., Matias, J., Jacob, E., \& Berbineau, M. (2008, September). The WiMAX ASN network in the V2I scenario. In Vehicular Technology Conference, 2008. VTC 2008-Fall. IEEE 68th (pp. 1-5). IEEE.

[2] Hameed, A. H., Mostafa, S. A., \& Mohammed, M. A. (2013). Simulation and evaluation of WIMAX handover over homogeneous and heterogeneous networks. American Journal of Networks and Communications, 2(3), 73-80.

[3] Hassan, M. H., Mostafa, S. A., Budiyono, A., Mustapha, A., \& Gunasekaran, S. S. (2018). A Hybrid Algorithm for Improving the Quality of Service in MANET. International Journal on Advanced Science, Engineering and Information Technology, 8(4), 1218-1225.

[4] Salih, A. I., Abdelouhahab, A., Mostafa, S. A., \& Zaiter, M. J. (2015) Updating the NCTUns-6.0 tool to simulate parallel optical burst switching of all-optical ultra-dense WDM systems. Photonic Network Communications, 29(1), 106-117. 
[5] Jubair, M. A., Mostafa, S. A., Mustapha, A., \& Gunasekaran, S. S. (2018). Performance Evaluation of Ad-Hoc On-Demand Distance Vector and Optimized Link State Routing Protocols in Mobile Ad-Hoc Networks. International Journal on Advanced Science, Engineering and Information Technology, 8(4), 1277-1283.

[6] Abdali, A. T. A. N., \& Muniyandi, R. C. (2017). Optimized Model for Energy Aware Location Aided Routing Protocol in MANET. International Journal of Applied Engineering Research, 12(14), 46314637.

[7] Khirbeet, A. S., \& Muniyandi, R. C. (2017). New Heuristic Model for Optimal CRC Polynomial. International Journal of Electrical and Computer Engineering (IJECE), 7(1), 521-525.

[8] Jubair, M., \& Muniyandi, R. (2016). NS2 Simulator to Evaluate the Effective of Nodes Number and Simulation Time on the Reactive Routing Protocols in MANET. International Journal of Applied Engineering Research, 11(23), 11394-11399.

[9] Mostafa, S. A., Tang, A. Y., Hassan, M. H., Jubair, M. A., \& Khaleefah, S. H. (2018, August). A Multi-Agent Ad Hoc On-Demand Distance Vector for Improving the Quality of Service in MANETs. In 2018 International Symposium on Agent, Multi-Agent Systems and Robotics (ISAMSR) (pp. 1-7). IEEE.

[10] Hassan, M. H., \& Muniyandi, R. C. (2017). An Improved Hybrid Technique for Energy and Delay Routing in Mobile Ad-Hoc Networks. International Journal of Applied Engineering Research, 12(1), 134-139.

[11] Al-Khaleefa, A. S., Ahmad, M. R., Muniyandi, R. C., Malik, R. F., \& Isa, A. A. M. (2018). Optimized Authentication for Wireless Body Area Network. Journal of Telecommunication, Electronic and Computer Engineering (JTEC), 10(2), 137-142.

[12] Halepovic, E., Ghaderi, M., \& Williamson, C. (2009, January). Multimedia application performance on a WiMAX network. In Multimedia Computing and Networking 2009 (Vol. 7253, p. 725309). International Society for Optics and Photonics.
[13] Adhicandra, I. (2010). Measuring data and VoIP traffic in WiMAX networks. arXiv preprint arXiv:1004.4583.

[14] Pentikousis, K., Piri, E., Pinola, J., Fitzek, F., Nissilä, T., \& Harjula, I. (2008, March). Empirical evaluation of VoIP aggregation over a fixed WiMAX testbed. In Proceedings of the 4th International Conference on Testbeds and research infrastructures for the development of networks \& communities (p. 19). ICST (Institute for Computer Sciences, SocialInformatics and Telecommunications Engineering).

[15] Haghani, E., \& Ansari, N. (2008, November). VoIP traffic scheduling in WiMAX networks. In Global Telecommunications Conference, 2008. IEEE GLOBECOM 2008. IEEE (pp. 1-5). Ieee.

[16] Sun, L., \& Ifeachor, E. C. (2006). Voice quality prediction models and their application in VoIP networks. IEEE Trans. Multimedia, 8(4), 809820.

[17] Carvalho, L., Mota, E., Aguiar, R., Lima, A. F., \& de Souza, J. N. (2005, June). An E-model implementation for speech quality evaluation in VoIP systems. In Computers and Communications, 2005. ISCC 2005. Proceedings. 10th IEEE Symposium on (pp. 933-938). IEEE.

[18] Sengupta, S., Chatterjee, M., \& Ganguly, S. (2008). Improving quality of VoIP streams over WiMax. IEEE transactions on computers, 57(2), 145-156.

[19] ITU, T. (2003). Recommendation G. 107 The E-model, a computational model for use in transmission planning.

[20] Jadhav, S., Zhang, H., \& Huang, Z. (2011, October). Performance evaluation of quality of VoIP in WiMAX and UMTS. In 2011 12th International Conference on Parallel and Distributed Computing, Applications and Technologies (pp. 375-380). IEEE.

[21] Chang, X. (1999, December). Network simulations with OPNET. In Proceedings of the 31st conference on Winter simulation: Simulation---a bridge to the future-Volume 1 (pp. 307-314). ACM. 